\title{
Interactive Segmentation of 3D UItrasound Using Deformable Solid Models and Active Contours
}

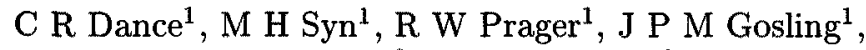 \\ L H Berman ${ }^{2}$ and K J Dalton ${ }^{3}$ \\ ${ }^{1}$ Cambridge University Engineering Dept., Trumpington Street \\ Cambridge CB2 1PZ, United Kingdom \\ ${ }^{2}$ Cambridge University Radiology Dept., Addenbrooke's Hospital \\ Cambridge CB2 2QQ, United Kingdom \\ ${ }^{3}$ Cambridge University Obstetrics \& Gynaecology Dept., Rosie Maternity \\ Hospital, Cambridge CB2 2QQ, United Kingdom
}

\begin{abstract}
This paper presents a prototype segmentation system for three-dimensional ultrasound data. 3D ultrasound is cheap and noninvasive but the data has a low signal-to-noise ratio and contains artifacts. To overcome these difficulties we have developed a system which uses a prior model, initialised by a clinician, to provide the starting point for a data-driven segmentation algorithm based on active contours. Results are presented showing how the technique can facilitate the segmentation of a gall-bladder.
\end{abstract}

\section{Introduction}

3D ultrasound is a new imaging modality with the potential to offer fully threedimensional visualisation and measurement of anatomical structures without the radiation required for CT or the cost of MRI. In order to assemble a 3D block of ultrasound data it is necessary to record the position and orientation of the scanning head when each set of echo responses are received. We use a Polhemus Fastrak magnetic sensor to measure the position and orientation of the probe on a conventional B-scan ultrasound machine. The resulting free-hand system provides an inexpensive way of recording $3 \mathrm{D}$ data of arbitrary shape and size. Furthermore, all the usual features of the B-scan machine such as a wide choice of probes and any Doppler facility can still be used.

There is a clinical requirement to measure changes in the volumes of various organs over time. Such data is used to monitor the progression of a disease or its response to treatment. For example, it is useful to measure the reduction in prostate size during reductase inhibitor therapy, and changes in splenic size during enzyme replacement therapy for Gaucher's disease. Current techniques for performing these measurements using ultrasound are either of limited accuracy or involve meticulous hand segmentation of the images which is very labour intensive.

Most current automatic segmentation techniques used in medical applications have been developed for CT or MRI images and perform badly with the 
high noise present in ultrasound. The free-hand 3D ultrasound data used in the present work can come in slices at any orientation. This means that the organ boundary in one slice will not always provide a good initial estimate for the segmentation of the next. Neither active contours, nor texture based segmentation will work automatically without clinical interaction to limit the search space and, where necessary, adjust the final solution. Model-based segmentation alone is not as accurate as a manual approach and requires initialisation by a clinician.

The novel contribution of this paper is to propose a hybrid approach making use of three sources of information: a deformable model [11], an active contour algorithm $[4,8]$ and a clinician. Initially, a solid deformable model is used to compute an approximate segmentation of the data. This is then used to initialise an active contour algorithm which runs interactively under the supervision of the clinician. Once each of the slices has been individually segmented the resulting contours are used to reconstruct the organ volume using Delaunay tetrahedranisation [4]. This strategy has the potential to greatly increase the speed at which a segmentation can be performed while maintaining the accuracy of the manual approach through interactive clinical supervision.

\section{Background}

A wide variety of automatic techniques have been applied to the segmentation of medical images [5] including thresholding and texture-based approaches, multi-scale techniques, region growing methods, model-based systems [6, 9] and algorithms based on snakes or active contours $[7,3,8]$. Combinations of these approaches have been explored and, for more difficult applications, semi-automatic systems have been developed in which the algorithms run interactively under the supervision of a clinician $[1,10]$.

As is mentioned in the previous section, ultrasound data is particularly difficult to segment because of the speckle and low signal-to-noise ratio [2]. In the case of free-hand 3D ultrasound we have the benefit of being able to relate each data point to its three dimensional environment, but there are also added complexities caused by the irregular multi-axial arrangement of the 2D slices in space.

\section{The Structure of the Segmentation System}

To address the difficulties listed above we have chosen to develop an interactive system, see figure 1. The clinician chooses the prior model and aligns it with the ultrasound data block by specifying correspondences between the two in $3 \mathrm{D}$ space. The computer deforms the model to fit the points specified by the clinician and computes the intersection of the model with each ultrasound slice. These intersections provide the starting point for an active contour based segmentation procedure which again involves the clinician. Where the angle between two image slices is small the initial contour can be projected from the previous slice that has been segmented [4]. At the start of the segmentation process, when the slices 


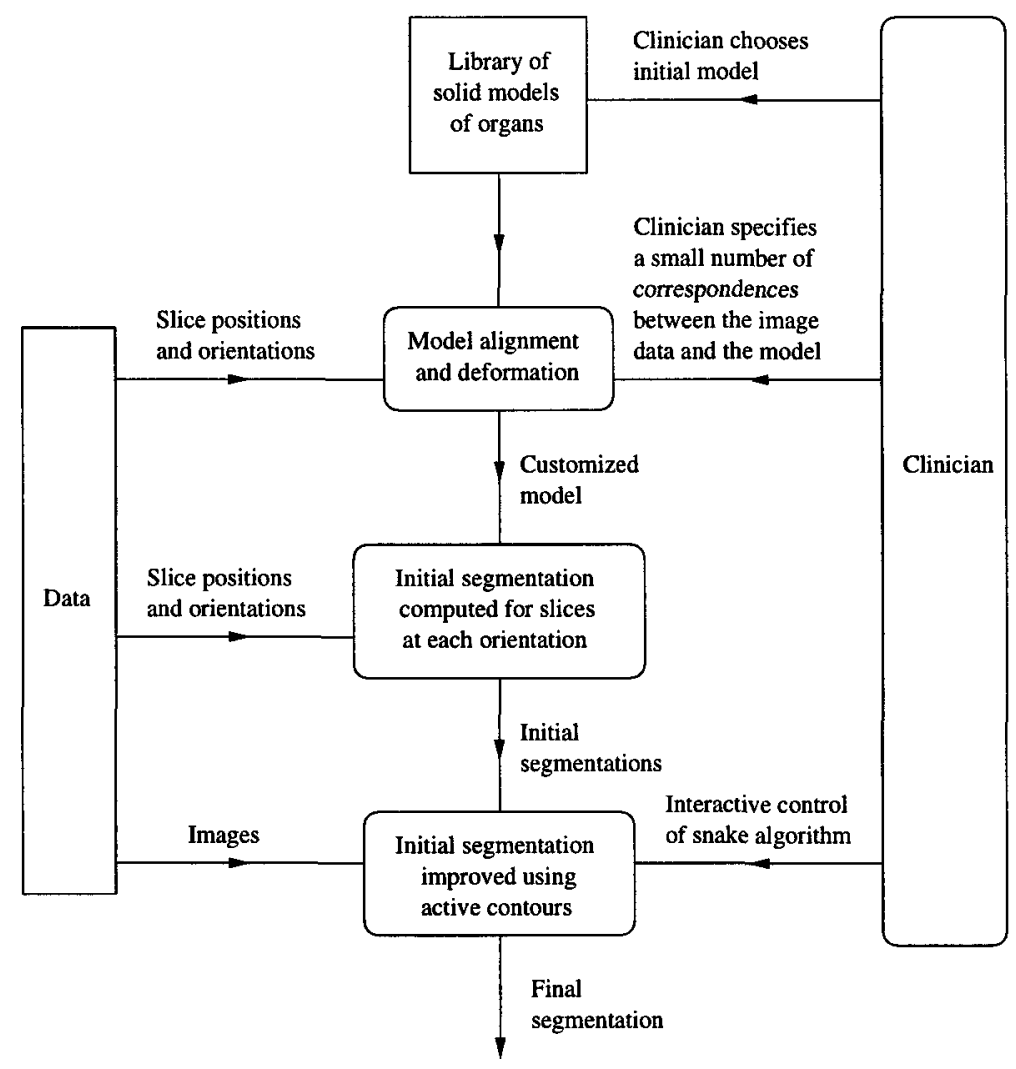

Figure 1: Block diagram of the segmentation system

move outside the organ, or when the slice angle changes significantly the trial contour is reinitialised from the model.

\section{Deformable Solid Models}

In this section the modes of deformation of organ shapes are derived by using the finite element method to compute numerical solutions to the wave equation. The organs are assumed to be homogeneous and linear-elastic under the deformations being considered.

The model is treated as an elastic body whose displacements at $n$ sample points are described by a vector $\mathbf{u}$. The elastic equilibrium implied by the wave equation then takes the form of a discretely sampled equilibrium system

$$
\mathbf{M} \ddot{\mathbf{u}}+\mathbf{K u}=\mathbf{0}
$$

which is of order $3 n$, since there are 3 displacement components at each sample point. In [11] we show how the body's mass matrix $\mathbf{M}$ and stiffness matrix $\mathbf{K}$ 
are assembled ${ }^{1}$.

Just as the wave equation can be expanded into eigenfunctions, the equilibrium equation (1) can be expanded into orthogonal FEM eigenmodes $\phi_{i}$, which are sampled and linearised approximations to the wave equation's eigenfunctions. First use separation of variables to solve equation (1)

$$
\mathbf{u}=\phi_{i} e^{j \omega_{\imath} t}
$$

where $\omega_{i}$ is the natural-frequency of vibration associated with $\phi_{i}$ (note that $j=\sqrt{-1}$ here). Applying this simple harmonic solution to equation (1) results in the eigenproblem

$$
\mathbf{K} \phi_{i}=\omega_{i}^{2} \mathbf{M} \phi_{i} .
$$

Given the mass matrix $\mathbf{M}$ and stiffness matrix $\mathbf{K}$ of an organ model, this eigenproblem is solved to give the eigenmodes $\phi_{i}$ and eigenvalues $\omega_{i}^{2}$. Eigenpairs $\left(\phi_{i}, \omega_{i}^{2}\right)$ are usually collected into the matrices $\left(\boldsymbol{\Phi}, \boldsymbol{\Omega}^{2}\right)$ and ranked in increasing order of eigenvalue

$$
\begin{aligned}
\boldsymbol{\Phi} & =\left(\phi_{1}, \phi_{2}, \ldots, \phi_{3 n}\right) \\
\boldsymbol{\Omega}^{2} & =\left(\begin{array}{cccc}
\omega_{1}^{2} & 0 & \cdots & 0 \\
0 & \omega_{2}^{2} & & \\
\vdots & & \ddots & \\
0 & & & \omega_{3 n}^{2}
\end{array}\right)
\end{aligned}
$$

By collecting eigenpairs into the matrices $\boldsymbol{\Phi}$ and $\boldsymbol{\Omega}^{2}$ equation (3) can be rewritten as

$$
\mathbf{K} \Phi=\mathbf{M} \Phi \boldsymbol{\Omega}^{2} .
$$

This is the generalised eigenproblem for the matrix pair $(\mathbf{M}, \mathbf{K})$. It occurs frequently in finite element analysis of structures, and there are powerful numerical techniques for computing $\boldsymbol{\Phi}$ and $\boldsymbol{\Omega}^{2}$.

Equation 6 shows how the model's deformation $\mathbf{u}$, can be described by projecting $\mathbf{u}$ onto the complete set of eigenmodes $\boldsymbol{\Phi}$. This approach is known as modal analysis, and is the natural approach to analysing structural deformation. The eigenmodes are an approximation to the elastic eigenfunctions, which describe the organ's shape variation.

\section{Active Contours}

Active contours or snakes are splines modelled as physical objects inhabiting some image space, which translate and deform under the action of a number of forces. In some cases deformation continues until the splines reach static equilibrium, but in others the motion of the spline is more relevant. In this work, closed or open piecewise linear splines (chains) are used to represent organ

\footnotetext{
${ }^{1} \mathbf{M}>\mathbf{0}$ and $\mathbf{K} \geq \mathbf{0}$ are both symmetric. $\mathbf{K}$ is positive semi-definite because an unsupported body can freely undergo rigid-body rotation and translation.
} 
boundaries within a B-scan plane. Two types of force are relevant; an internal or unbending force which encourages smooth contour shapes, and an external or image force which encourages the contour to be located in regions which are heterogeneous with respect to some feature value. The external force may be described by a potential but the internal force cannot be. Additionally, vertices of the chain may be constrained to lie in fixed position rather than moving under the influence of the forces.

By analogy with physics, a contour is given a dynamic state. The net force acting on each vertex produces a rate of change of its velocity $\mathbf{v}_{\mathbf{k}}$. This force is taken to be a weighted sum of the internal and external forces described above combined with a dissipative damping force serving to reduce oscillations. The scheme used in the present work is adapted from [8] and fully described in [4]. Deformation may be paused and contours manually shifted to correct for segmentation errors. Termination of deformation is also manually controlled.

\section{The Segmentation System in Action}

We now describe the complete segmentation process and illustrate it using data from a $3 \mathrm{D}$ scan of a gall-bladder. The process begins with a set of twodimensional ultrasound scans and a mathematical model of the organ to be segmented.

Figure 2(a) shows a typical 2D scan, figure 2(b) shows the positions of all these scans in the $3 \mathrm{D}$ data-set. A symbol like 2(c) is drawn to indicate the position of each scan plane. In 2(c) the scan planes are viewed edge-on and therefore appear almost as straight lines.

A uniformly tessellated ellipsoid is chosen as the prior model. The seventh eigenmode of this solid model is shown in figure 3. The first six eigenmodes are rigid-body transformations of translation and rotation. This figure shows the simplest model distortions which are available to make the model fit the data.

The clinician chooses a scan plane that passes longitudinally through the middle of the gall-bladder and, using the graphical user interface, specifies a small number of correspondences between points on the scan plane and points on the model. Four correspondences will establish a match making use of the first seven eigenmodes of the model. More points could be chosen, using more than one scan plane, and a match would be performed using higher order modes. In this example we use just four points, see figure 4.

The model is aligned with the data using the four correspondences, then scaled and deformed to fit. The accuracy of the fit is determined by a stiffness parameter in the algorithm which controls the degree of distortion.

Now that the data and the model are aligned, the intersection of the model with any scan plane can be computed and used as an initial segmentation. The main benefit of the current approach becomes apparent when we come to segment ultrasound slices taken in different directions than the original plane in which the correspondences were specified. In these cases, simpler methods of automatically 


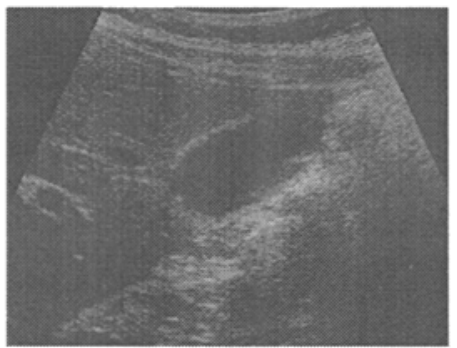

(a) An example 2D scan

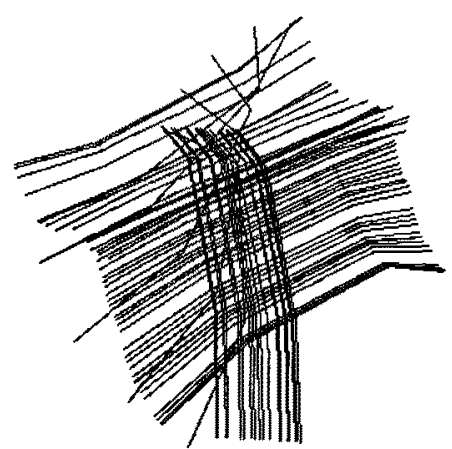

(b) Positions of $2 \mathrm{D}$ scans

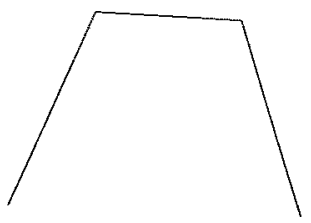

(c) Outline used to show $2 \mathrm{D}$ scan position

Figure 2: Format of the 3D ultrasound data

initialising the trial contour fail but the intersection with the model is still easy to compute. Figure 5 shows the results from one such plane.

\section{Discussion and Conclusions}

We have presented a new semi-automatic segmentation system for $3 \mathrm{D}$ ultrasound that uses deformable solid models to initialise an active contour algorithm. This combination of a top-down model-based approach with a data-driven contour segmentation algorithm enables us to reduce the effort required from the clinician to perform the mechanical repetitive parts of the segmentation while retaining clinical involvement in the key aspects of the process that ensure an accurate end result.

\section{References}

[1] W.A. Barrett and E.N. Mortensen. Fast, accurate and reproducible live-wire boundary extraction. In K.H. Höhne and Kikinis R., editors, Proceedings of the 4th International Conference on Visualization in Biomedical Computing, volume 1131 of Lecture Notes in Computer Science, pages 183-192. Springer, September 1996. 

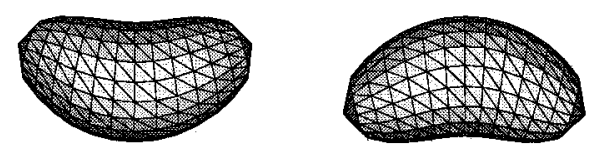

Figure 3: 7th eigenmode of a uniformly tessellated ellipsoid

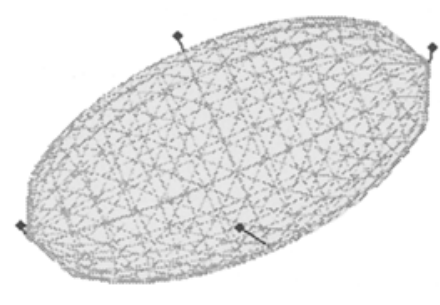

(a) Points on the model

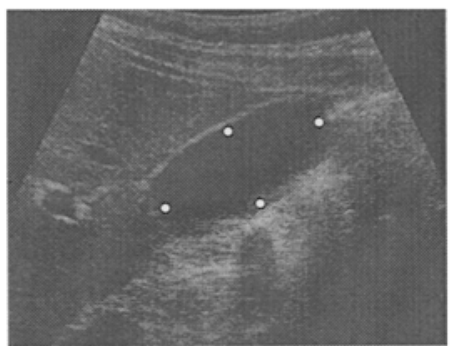

(b) Points on the image

Figure 4: Correspondences between the model and the 3D data set

[2] C.J. Bouma, W.J. Niessen, K.J. Zuiderveld, E.J. Gussenhoven, and M.A. Viergever. Evaluation of segmentation algorithms for intravascular ultrasound images. In Proceedings of the 4 th International Conference on Visualization in Biomedical Computing, pages 203-212, September 1996.

[3] L.D. Cohen and I. Cohen. Finite-element methods for active contour models and balloons for 2-d and 3-d images. IEEE Transactions on Pattern Analysis and Machine Intelligence, 15(11):1131-1147, 1993.

[4] C.R. Dance. Computing models from $3 D$ ultrasound. PhD thesis, Cambridge University, 1997.

[5] R.M. Haralick and L.G. Shapiro. Image segmentation techniques. Computer Vision, Graphics and Image Processing, 29:100-132, 1985.

[6] A. Hill, A. Thornham, and C.J. Taylor. Model based interpretation of 3d medical images. In Proceedings of the British Machine Vision Conference, pages 339-348, 1993.

[7] M. Kass, A. Witkin, and D. Terzopoulos. Snakes: active contour models. In Proc. Ist International Conference on Computer Vision, pages 259-268, 1987.

[8] A. Lobregt and M.A. Viergever. A discrete dynamic contour model. IEEE Transactions on Medical Imaging, 14(1):12-24, 1995. 


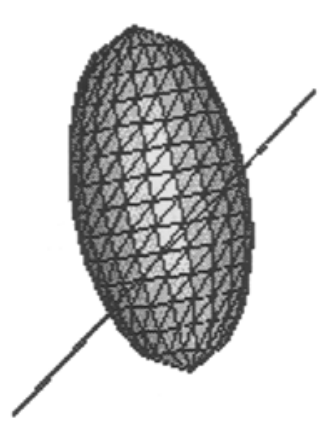

(a) Relation of scan plane to model

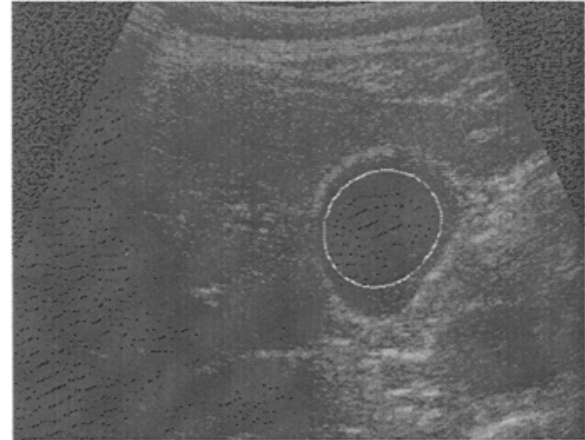

(b) Intersection of model with scan plane

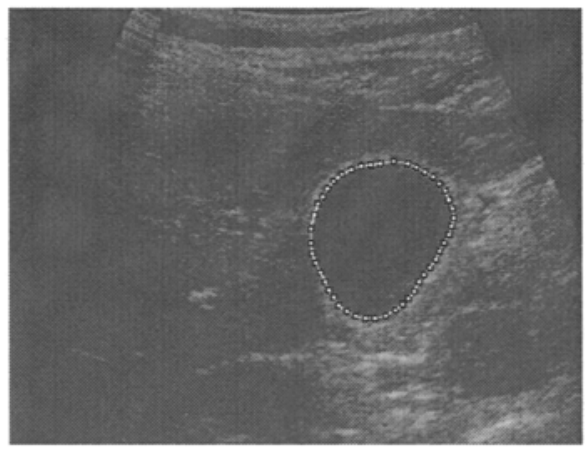

(c) Improved segmentation using active contour

Figure 5: Segmentation of an image intersecting the model in a different direction to that in which the correspondences were specified

[9] A. Pentland and S. Sclaroff. Closed-form solutions for physically based shape modeling and recognition. IEEE Trans. Pattern Analysis and Machine Intelligence, 13(7):715-729, 1991.

[10] S. Revankar, D. Sher, C. Cheung, V.L. Shalin, M. Ramamurthy, and S. Rosenthal. Supervised interpretation of echocardiograms with a psychological model of expert supervision. Computerized Medical Imaging and Graphics, 19(1):47-59, 1995.

[11] M.H. Syn. Model-based three-dimensional freehand ultrasound imaging. PhD thesis, Cambridge University, 1996. 